\title{
An Implantable Wireless Multi-Channel Neural Prosthesis for Epidural Stimulation
}

\author{
Dai Jiang ${ }^{1}$, Clemens Eder ${ }^{1}$, Timothy A. Perkins ${ }^{2}$, Anne Vanhoestenberghe ${ }^{3}$, Matthew Schormans ${ }^{1}$, Fangqi Liu ${ }^{1}$, \\ Virgilio Valente ${ }^{1}$, Nick Donaldson ${ }^{2}$, and Andreas Demosthenous ${ }^{1}$ \\ ${ }^{1}$ Department of Electronic and Electrical Engineering, ${ }^{2}$ Department of Medical Physics and Bioengineering, ${ }^{3}$ Institute of \\ Orthopaedics and Musculoskeletal Sciences, University College London, London WC1E 6BT, United Kingdom \\ d.jiang@ucl.ac.uk, a.demosthenous@ucl.ac.uk
}

\begin{abstract}
This paper presents a fully implantable multi-channel neural prosthesis for epidural stimulation. The prosthesis features three telemetry-operated independent stimulators providing in total eighteen stimulation channels. The stimulator circuits were implemented in a $0.6-\mu \mathrm{m}$ CMOS technology. The prosthesis is protected in a hermetically sealed ceramic enclosure and encapsulated in medical grade silicone rubber. In-vitro measured results with electrodes in saline are presented.
\end{abstract}

\section{INTRODUCTION}

Research has demonstrated the potential of using epidural stimulation to restore coordinated locomotion, such as standing and walking, to patients suffering from paralysis due to severe spinal cord injury (SCI) [1]-[4]. As illustrated in Fig. 1, in the absence of input from the brain as a result of lesion in the spinal cord, the natural sensory information required for locomotion is projected onto the spinal cord by applying modulated electrical stimuli via an epidural electrode array implanted along the spinal cord. The intrinsic sensorimotor neural circuits embedded in the spinal cord can generate organised motor patterns for standing, walking and climbing in response to the stimulation. Research in animal models has shown a linear relationship between the frequency or amplitude of the electrical stimuli in pulsatile stimulation and the step height. A neural modulation scheme developed based on this result has enabled rats to walk on a treadmill for 1000 steps without failure [4]. Clinical trials in several human subjects also achieved weight-bearing standing and assisted stepping behaviour under epidural stimulation [1][3].

Advances in the epidural stimulation research demand a purpose built, multi-mode stimulator that is able through the implantable multi-electrode array to selectively target multiple sites of the spinal circuitries with precisely controlled pulse timing and intensity [4]-[5]. In addition, the stimulator should support simultaneous stimulation in multiple channels with advanced neural modulation schemes to coordinate variable motor outputs into unique functional movement such as standing and stepping [6]. The stimulator should also be suitable for chronic implantation for long-term studies. The stimulators used in existing studies, are either external devices with percutaneous

This work was funded by the European Commission under grant number 258654 - NEUWalk (www.neuwalk.eu).

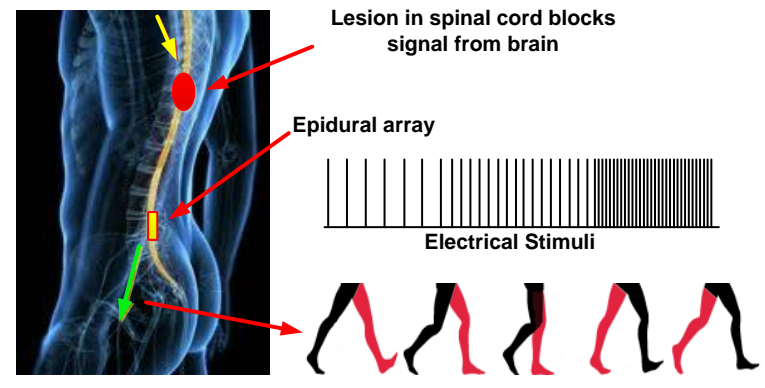

Fig. 1. Epidural stimulation facilitates gait cycles by modulating the electrical stimuli.

connections to implanted electrodes [4], [5], [7] that are only suitable for acute study, or modified commercial medical implants for off label use that only support simple on-off neural modulation [1]-[3].

A wireless, fully implantable, multi-site, multi-mode epidural prosthesis is presented which has three independent stimulators providing uncorrelated simultaneous stimulation in similar or different modulation schemes, targeting multiple sites along the spinal cord. Each stimulator supports multiple sites for selecting the most effective "hot spots". The prosthesis is hermetically sealed in a ceramic package and encapsulated for chronic implantation. The implant is wirelessly powered by an inductive link and is controlled by a remote (programming) computer over a $900 \mathrm{MHz}$ radio link, primarily for research studies with freely moving animals. The rest of the paper is organised as follows. Section II describes the overall architecture of the neural prosthesis, the operation of the telemetry, the stimulation procedure and the implant packaging. Section III presents measured results from in-vitro tests, followed by conclusions in Section IV.

\section{SySTEM AND CIRCUIT ARCHITECTURE}

\section{A. Overall System}

The architecture of the implantable prosthesis system is shown in Fig. 2(a). The system comprises a wearable transmitter, an implantable prosthesis and a remote host computer. The 

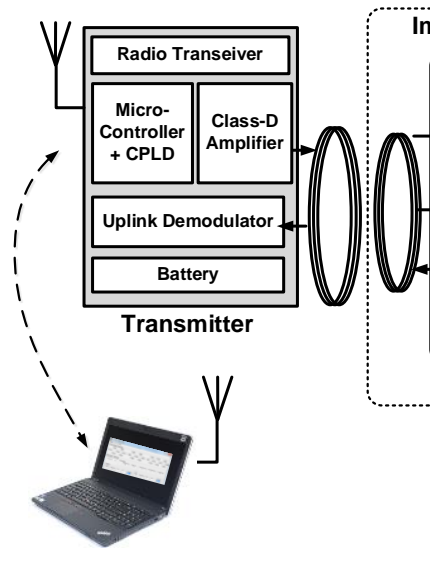

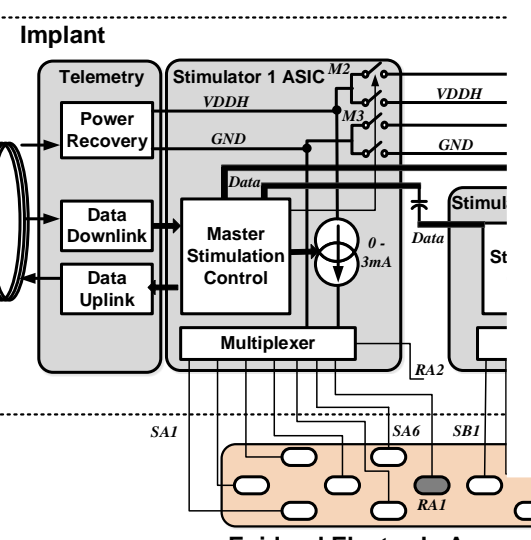

Epidural Electrode Array

(a)

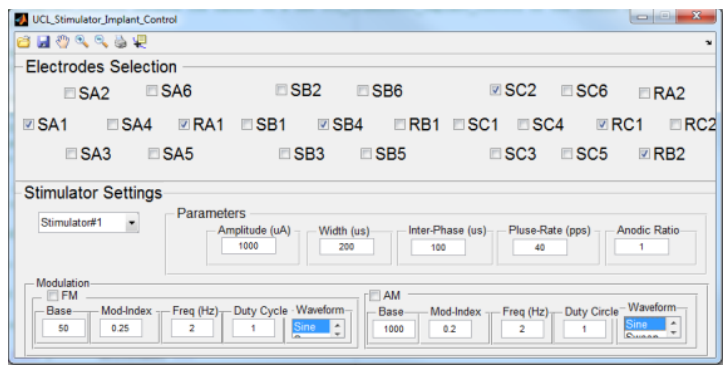

Fig. 4. Graphical user interface (GUI) for setting stimulation parameters.

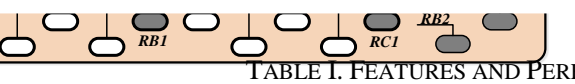

\begin{tabular}{l|l}
\hline Hybrid dimensions & $46 \mathrm{~mm} \times 42.8 \mathrm{~mm} \times 8.8 \mathrm{~mm}$ \\
\hline Stim
\end{tabular}

\begin{tabular}{l|l} 
Stimulator ASICs & $0.6-\mu \mathrm{m} \mathrm{HV} \mathrm{CMOS}$ \\
& $(b)$
\end{tabular}

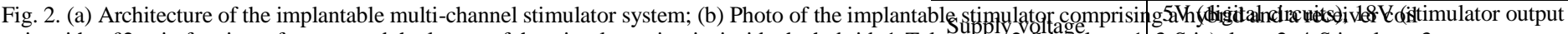
unit, with a $£ 2$ coin for size reference, and the layout of the stimulator circuits inside the hybrid: 1-Telemetry, 2-Stimulator 1, 3stsigenulator 2, 4-Stimulator 3.

wearable transmitter receives instructions from the remote host via a $900 \mathrm{MHz}$ radio link and forwards these instructions to the implant via a $9.6 \mathrm{MHz}$ inductive link. A class-D amplifier in the transmitter drives the primary coil of the inductive link to power the implant. The inductive link also serves as a bi-directional half-duplex communication link. The processor in the transmitter translates received instructions into command frames that are sent to the implant for setting the stimulation characteristics. The frames are sent via downlink transmission whose carrier is modulated by on-off keying (OOK) at 400 kbit/s. The uplink acknowledgement from the implant to the transmitter uses passive-phase-shift keying (PPSK) modulation with a data rate at $600 \mathrm{kbit} / \mathrm{s}$.

The implantable prosthesis comprises a receiver coil unit and the hybrid, as shown in Fig. 2(b), connected with Cooner Wire AS632. The receiver coil unit consists of only passive components, including the secondary coil of the inductive link and half-wave rectifiers to convert received power into dc voltages. There is a rare earth magnet at the center of the receiver coil unit for alignment with another magnet in the primary coil, also located its center. The hybrid comprises a telemetry for power regulation and data communication, and three parallel integrated stimulators (ASICs), as shown in Fig. 2(a) and 2(b). Each stimulator drives six stimulating electrodes (SA1-SA6, SB1$S B 6$ and SC1-SC6) and two return electrodes (RA1-RA2, RB1$R B 2$ and $R C 1-R C 2$ ) on the epidural electrode array. A stimulation channel is formed with one stimulating electrode and one return electrode. In total eighteen channels can be formed between the three stimulators.

\section{B. Implant Electronics}

The implant electronics are separated into the receiver coil unit and the hybrid. As shown in Fig. 3, the receiver coil unit has six connections to the hybrid, including a high voltage supply, a low voltage supply, the ground $(0 \mathrm{~V})$ rail, the carrier frequency as a $9.6 \mathrm{MHz}$ clock, and two communication ports for the uplink and downlink data. The secondary coil, $L_{2}$, is tuned with $C_{2}$ at 9.6 $\mathrm{MHz}$. The coupled oscillating signal on $L_{2} C_{2}$ is rectified by $D_{5} C_{4}$

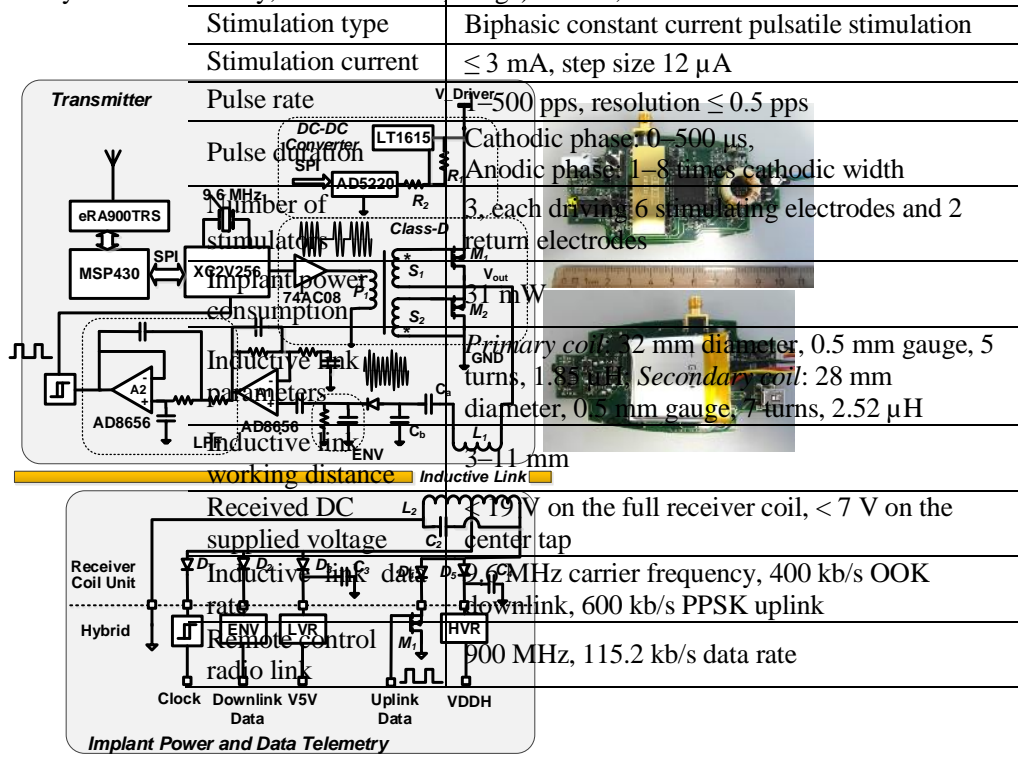

Fig. 3. Schematic of the power and data telemetry, with photos of the external transmitter (top and bottom sides).

into a dc voltage, which provides a stable $18 \mathrm{~V}$ supply using a high voltage regulator (HVR, MIC5233) in the hybrid. Another half-wave rectifier $D_{3} C_{3}$ is connected to the centre tap on $L_{2}$ to extract a lower dc voltage, which is regulated by a low voltage regulator (LVR) in the hybrid to a $5 \mathrm{~V}$ supply for the stimulation control logic. The LVR is implemented in the Stimulator 1 ASIC. An envelope detector (ENV) implemented in the Stimulator 1 ASIC in the hybrid is connected via $D_{2}$ to the centre tap on $L_{2}$ to demodulate the downlink data in the OOK scheme. The signal on the centre tap is also connected via $D_{l}$ to a Schmitt trigger (74AHCT1G14) in the hybrid to serve as a RF clock for the uplink data modulation (using PPSK [8]). In this scheme, the control logic in Stimulator 1 ASIC shorts $L_{2}$ by turning on a transistor switch, $M_{l}$, also implemented in the same ASIC, for exactly $1.5 \mathrm{RF}$ clock cycles from the time instant when the voltage on $L_{2}$ crosses zero. This action causes a transient voltage surge on the primary coil, $L_{l}$. Uplink data transfer is achieved by applying the uplink bit-stream to the gate of $M_{l}$ at a speed of 600 
kbit/s. Similar to load-shift keying (LSK), PPSK is also a passive modulation scheme but provides a faster data rate because of its transient nature.

The hybrid derives both high and low dc voltages as well as the downlink data from the receiver coil unit for the operation of the three parallel stimulators. The circuits of the stimulators are integrated into three ASICs. Each ASIC comprises a control logic, a $5 \mathrm{~V}$ regulator to generate a stable dc voltage for the logic, a 8-bit linear current digital-to-analog converter (DAC) with a dynamic range between $0 \mathrm{~mA}$ and $3 \mathrm{~mA}$, an " $\mathrm{H}$ "-bridge output stage to form biphasic current pulses, and a multiplexer to select the stimulating and return electrodes. Stimulator 1 ASIC also contains demodulator circuits for the downlink and a modulator for the uplink as previously described. The control logic in the Stimulator 1 ASIC also handles the bi-directional communication with the transmitter and manages the stimulation settings on all the three stimulators. A power-isolation method [9] was implemented to provide efficient isolation between stimulators, so that the crosstalk between the stimulators is minimised and thus ensuring the independence of the modulation scheme and pulse timing on each stimulator. In this arrangement, the power supplies to Stimulator ASIC 2 and 3 are gated in Stimulator ASIC 1, as shown in Fig. 2(a). During a biphasic pulse on ASIC 2 or ASIC 3, the switches to the ASIC (M2 or M3) are switched off and the ASIC is supplied by the storage capacitor, $C 2$ or $C 3$, respectively. After the current pulse, the ASIC is reconnected to the power supply for charging the storage capacitor. Stimulation settings to ASIC 2 and ASIC 3 are sent via a capacitive data link from Stimulator ASIC 1. This power isolation ensures no potential crosstalk paths so that current pulses can occur simultaneously on multiple stimulators without interfering with each other.

\section{Implant Packaging and Encapsulation}

The components in the hybrid were mounted on a ceramic (96\% alumina) substrate in a size of $36 \mathrm{~mm} \times 38 \mathrm{~mm}$ with gold and platinum-gold tracks thick-film printed. The circuits were hermetically sealed under a metallised ceramic lid, as shown in Fig. 2(b). Six contacts to the receiver coil unit and twenty-four contacts to the epidural electrode array outside the lid were connected to the circuits via hermetic feedthroughs. The components in the receiver coil unit were mounted on a FR4 printed circuit board. The two units were joined by Cooner wires. The whole implant was encapsulated in medical grade silicone rubber EPM2420.

\section{External Transmitter and Remote Control}

The hardware architecture of the transmitter is shown in Fig. 3. The operation of the transmitter is managed by a microcontroller (MSP430). The microcontroller communicates with a remote computer via a $900 \mathrm{MHz}$ radio link implemented with easyRadio advanced ISM radio module eRA900TRS [10]. Stimulation parameters, including the pulse settings and the modulation schemes, are defined through a graphical user interface, shown in Fig. 4, and sent to the microcontroller via the radio link. The settings are then be passed to a CPLD device $\mathrm{XC} 2 \mathrm{~V} 256$, where command frames are formed with additional

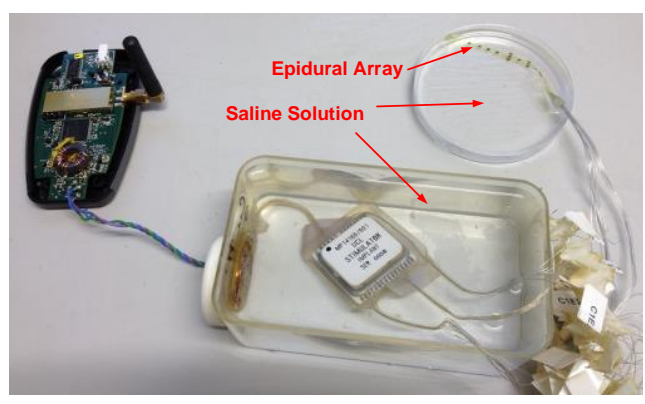

Fig.5. Setup for in-vitro testing.

CRC bits. The CPLD also applies OOK modulation to a $9.6 \mathrm{MHz}$ carrier. A class-D amplifier drives the primary coil, $L_{1}$, with the modulated carrier signal to power the implant. The class-D amplifier is powered from a dc-dc converter LT1615, whose output voltage is programmed by the microcontroller via a potentiometer AD5220. According to the feedback from the implant, the transferred power is adjusted to improve power efficiency. The uplink data are demodulated from the voltage on $C_{b}$, which together with $C_{a}$ tunes $L_{l}$ to $9.6 \mathrm{MHz}$. The voltage surge on the carrier is extracted with a low-pass filter (LPF, built with Op-Amp A2, AD8656) preceded by an envelope detector (ENV) and an amplifier (built with Op-Amp A1, AD8656). The demodulated bit-stream is then converted to digital by a Schmitt trigger (74LVC1G17). The CPLD extracts information from the bit-stream and passes it to the microcontroller. The transmitter is supplied by a rechargeable lithium polymer battery LP-443440 (3.7 V, $600 \mathrm{mAh})$.

\section{MEASURMENTS}

The stimulator ASICs were fabricated in a $0.6-\mu \mathrm{m}$ highvoltage CMOS technology. The stimulators used separate chips to prevent a common substrate that would prevent power isolation. The implant features and the measured specifications are summarised in Table I.

The neural prosthesis system was tested in-vitro as shown in Fig. 5. The implant was submerged in saline solution (16.7 $\mathrm{mS} / \mathrm{cm}^{2}$ ). The secondary coil in the implant is aligned with the primary coil through the wall of the container. The implant is connected to an epidural electrode array (Courtesy of Fraunhofer ICT-IMM) that is also submerged in saline solution. The connection was made at an adaptor for measuring the current pulses. Fig. 6 shows the performance of alternative stimulations on multiple channels from a single stimulator. Three electrodes on an array were connected to three stimulating outputs on Stimulator 1. Symmetrical biphasic pulses with amplitude of 800 $\mu \mathrm{A}$ and a pulse rate of $100 \mathrm{pps}$ were delivered to the three electrodes in a sequence where, in a cycle with a length of one second, stimuli on Electrode $S A 1$ started from $0 \mathrm{~s}$ to $0.4 \mathrm{~s}$, on Electrode $S A 2$ from 0.3 s to $0.7 \mathrm{~s}$ and on Electrode $S A 3$ from 0.5 $\mathrm{s}$ to $0.9 \mathrm{~s}$. Fig. 7 demonstrates independent modulation schemes on different stimulators. On Stimulator 1, $2 \mathrm{~Hz}$ sinusoidal amplitude modulation was applied with a base amplitude of 300 $\mu \mathrm{A}$ and a swing of $400 \mu \mathrm{A}$. On Stimulator 2 and Stimulator 3, 2 $\mathrm{Hz}$ sinusoidal frequency modulation was applied with a base 


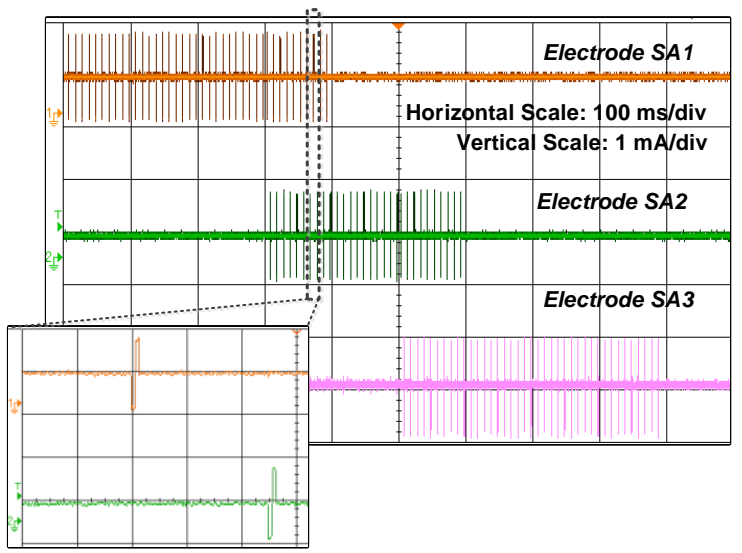

Fig. 6. Measured waveforms of an exemplar interleaved pulses from one stimulator for sequentially stimulating multiple "hot spots".

pulse rate of $250 \mathrm{pps}$ and a swing of $400 \mathrm{pps}$.

\section{CONCLUSIONS}

A wirelessly powered and controlled, fully implantable, multi-channel, multi-mode neural prosthesis for epidural spinal cord stimulation has been presented. The prosthesis has been developed for research on restoring locomotion to patients after spinal cord injury. The details of the system implementation have been presented, and the functionality has been demonstrated with in-vitro tests.

\section{ACKNOWLEDGEMENT}

The authors would like to thank Prof David Borton for the discussions on the device specifications and Dr Peter Detemple for providing the epidural electrode array for the in-vitro evaluation.

\section{REFERENCES}

[1] S. Harkema, Y. Gerasimenko, J. Hodes, J. Burdick, C. Angeli, et al., "Effect of epidural stimulation of the lumbosacral spinal cord on voluntary movement, standing, and assisted stepping after motor complete paraplegia: a case study," Lancet, vol. 377, pp. 1938-1947, 2011.

[2] D. G. Sayenko, C. Angeli, S. J. Harkema, V. R. Edgerton, and Y. P. Gerasimenko, "Neuromodulation of evoked muscle potentials induced by epidural spinal-cord stimulation in paralyzed individuals," J. Neurophysiol., vol. 111, pp. 1088-1099, 2014.

[3] C. A. Angeli, V. R. Edgerton, Y. P. Gerasimenko, and S. J. Harkema, "Altering spinal cord excitability enables voluntary movements after chronic complete paralysis in humans," Brain, vol. 137, pp. 1394-1409, 2014.

[4] N. Wenger, E. M. Moraud, S. Raspopovic, M. Bonizzato, J. DiGiovanna, P. Musienko, M. Morari, and S. Micera, G. Courtine, "Closed-loop neuromodulation of spinal sensorimotor circuits controls refined locomotion after complete spinal cord injury," Sci. Transl. Med., vol. 6, p. 255ra133, 2014.

[5] P. Gad, J. Choe, M. S. Nandra, H. Zhong, R. R. Roy, Y. C. Tai, and V. R. Edgerton, "Development of a multi-electrode array for spinal cord epidural stimulation to facilitate stepping and standing after a complete spinal cord injury in adult rats," J. Neuroeng Rehabil., 10:2, 2013.

[6] D. Borton, M. Bonizzato, J. Beauparlant, J. DiGiovanna, E. M. Moraud, N. Wenger, P. Musienko, I. R. Minev, S. P. Lacour, R. Millán Jdel, S. Micera, and G. Courtine, "Corticospinal neuroprostheses to restore locomotion after spinal cord injury," Neurosci. Res., vol. 78, pp. 21-29, 2014.

[7] R. van den Brand, J. Heutschi, Q. Barraud, et al., "Restoring voluntary control of locomotion after paralyzing spinal cord injury," Science, vol. 336, pp. $1182-1185,2013$.

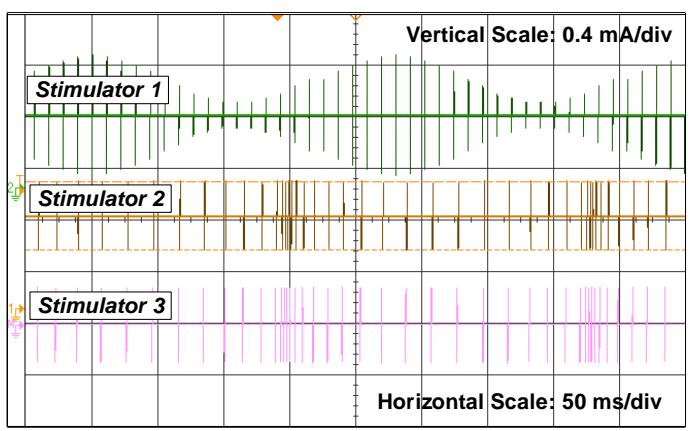

Fig. 7. Measured waveforms of independent stimulation from the three stimulators with different modulation schemes.

[8] D. Cirmirakis, D. Jiang, A. Demosthenous, N. Donaldson, and T. Perkins, "A fast passive phase shift keying modulator for inductively coupled implanted medical devices," Proc. ESSCIRC 2012, pp. 301-304.

[9] D. Jiang, D. Cirmirakis, and A. Demosthenous, "A vestibular prosthesis with highly-isolated parallel multichannel stimulation," IEEE Trans. Biomed. Circuits Syst. Vol. 9, pp. 124-137, 2015.

[10] LPRS, eRA900TRS, http://www.lprs.co.uk/assets/media/easyRadio\%20 Advanced\%20Datasheet\%20v3.6.pdf 